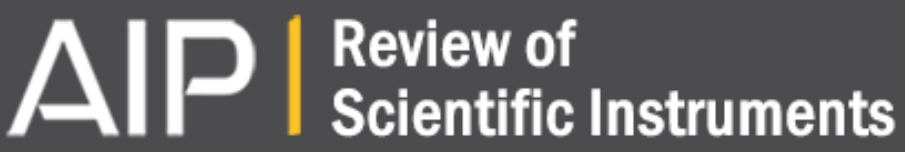

A compact instrument for adjusting laser beams to be accurately coincident and coaxial and its use in biomedical imaging using wave-mixed laser sources

R. Amor, G. Norris, J. Dempster, W. B. Amos, and G. McConnell

Citation: Review of Scientific Instruments 83, 083705 (2012); doi: 10.1063/1.4745373

View online: http://dx.doi.org/10.1063/1.4745373

View Table of Contents: http://scitation.aip.org/content/aip/journal/rsi/83/8?ver=pdfcov

Published by the AIP Publishing

\section{Articles you may be interested in}

Two-photon excitation microscopy using the second singlet state of fluorescent agents within the "tissue optical window"

J. Appl. Phys. 114, 153102 (2013); 10.1063/1.4825319

Preliminary investigations on the determination of three-dimensional dose distributions using scintillator blocks and optical tomography

Med. Phys. 40, 082104 (2013); 10.1118/1.4813898

Ultrafast optics: Imaging and manipulating biological systems

J. Appl. Phys. 105, 051101 (2009); 10.1063/1.3081635

Microbeam-integrated multiphoton imaging system

Rev. Sci. Instrum. 79, 123707 (2008); 10.1063/1.3043439

Diode-pumped solid state laser light sources for confocal laser scanning fluorescence microscopy

J. Laser Appl. 20, 160 (2008); 10.2351/1.2955554

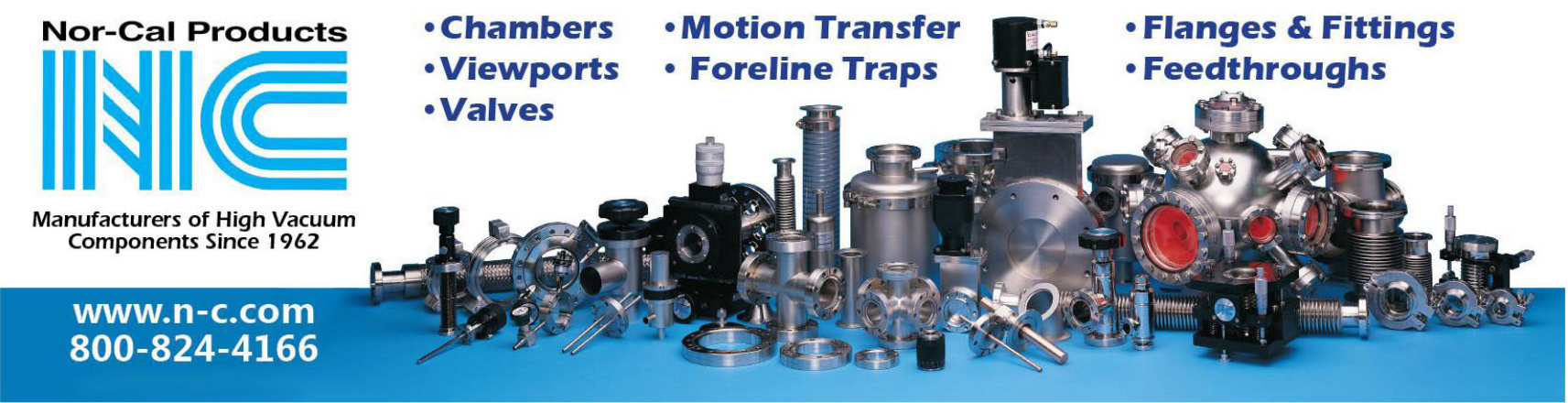




\title{
A compact instrument for adjusting laser beams to be accurately coincident and coaxial and its use in biomedical imaging using wave-mixed laser sources
}

\author{
R. Amor, ${ }^{1, \text { a) }}$ G. Norris, ${ }^{1}$ J. Dempster, ${ }^{1}$ W. B. Amos, ${ }^{2}$ and G. McConnell ${ }^{1}$ \\ ${ }^{1}$ Centre for Biophotonics, Strathclyde Institute of Pharmacy and Biomedical Sciences, University \\ of Strathclyde, 161 Cathedral Street, Glasgow G4 ORE, United Kingdom \\ ${ }^{2}$ MRC Laboratory of Molecular Biology, Hills Road, Cambridge CB2 2QH, United Kingdom
}

(Received 27 March 2012; accepted 26 July 2012; published online 16 August 2012)

\begin{abstract}
Biomedical imaging applications that involve nonlinear optical processes such as sum-frequency generation (SFG) and four-wave mixing require that the pulses are synchronized in time and the beams are coaxial to better than $400 \mu \mathrm{rad}$. For this reason, folding mirrors are normally used to extend the beam path over a few meters so that detectors can be put into the beams to check their overlap at the start of a long path and also at the end of it. We have made a portable instrument with a footprint of only $22 \mathrm{~cm} \times 11 \mathrm{~cm} \times 16 \mathrm{~cm}$ that uses a short focal length lens and a telephoto combination for viewing the near-field and far-field simultaneously. Our instrument is simple to build and use, and we show its application in coherent anti-Stokes Raman scattering microscopy and SFG-based two-photon fluorescence microscopy. (C) 2012 American Institute of Physics. [http://dx.doi.org/10.1063/1.4745373]
\end{abstract}

\section{INTRODUCTION}

Current optical microscopy techniques for biomedical imaging routinely make use of the nonlinear process of wavemixing to either provide the excitation beam or to excite the specimen to produce the optical signal.

One such technique is coherent anti-Stokes Raman scattering (CARS) microscopy, a four-wave mixing process in which a pump beam, a Stokes beam, and a probe beam interact with the specimen and generate an anti-Stokes beam. This anti-Stokes beam is the CARS signal, and when the difference frequency between the pump and Stokes beams coincides with a molecular vibration within the specimen, this signal is greatly enhanced, making real-time imaging possible. ${ }^{1-6}$

Another optical microscopy technique that makes use of wave-mixing is sum-frequency generation (SFG)based two-photon fluorescence microscopy. Traditionally, the femtosecond-pulsed Ti:sapphire laser is the excitation source for two-photon fluorescence imaging. ${ }^{7}$ This laser provides an average power in excess of $1 \mathrm{~W}$ and is wavelength-tunable in the range of 690-1050 nm, which makes it ideal for imaging a wide range of endogenous and synthetic fluorophores. However, there are fluorophores such as $\mathrm{NAD}(\mathrm{P}) \mathrm{H}$ and flavoprotein that have peak two-photon excitation wavelengths deep into the ultraviolet that are not accessible by the Ti:sapphire laser. $^{8}$ In order to perform two-photon fluorescence imaging on these molecules, non-traditional laser sources are required and one route towards achieving this is by using SFG to provide a sub-700 $\mathrm{nm}$ excitation source for imaging in the ultraviolet range.

The four-wave mixing nature of CARS microscopy and the three-wave mixing nature of SFG-based two-photon fluorescence microscopy mean that both are multiphoton processes. This dictates that the strength of the generated signal is directly dependent on the temporal and spatial overlap of

a)Electronic mail: rumelo.c.amor@strath.ac.uk. the incident pulses. ${ }^{9-11}$ These techniques therefore require the standard method of achieving temporal overlap between two pulses, which is through optimization of the SFG output of the beams through a nonlinear crystal. ${ }^{12}$ In the case of spatial overlap, the experimental setup normally incorporates a series of folding mirrors for evaluating beam overlap both in the near-field and far-field. ${ }^{13,14}$ The folded beam occupies much additional space in a laser system that is already large and the long path length exposes the beam to thermal and mechanical disturbance, which can negatively affect the performance of the microscope.

We have devised a compact optoelectronic instrument to replace the folded path to visualize spatial beam overlap. Using a lens of short focal length as a microscope and a telephoto combination of large focal length as a telescope, both forming images of the beam cross-sections in the same camera, we are able to view the beams in the near-field and far-field simultaneously, thus eliminating the need for switching between near-field and far-field views and saving space on the optical table and reducing the likelihood of eye or skin exposure to the laser beams.

\section{EXPERIMENT}

A key component of our instrument is a $50 \%$ beamsplitter (25 BI 00, Comar Instruments, UK) which divides the beams into near-field and far-field components, allowing a microscopic image of both beam cross-sections to be formed at a near position and a telescope image to be formed at an infinite distance, as shown in Fig. 1. The effective focal length of the combination of a positive and negative lens is given by the equation: ${ }^{15}$

$$
\frac{1}{f_{e}}=\frac{1}{f_{p}}+\frac{1}{f_{n}}-\frac{d}{f_{p} f_{n}},
$$




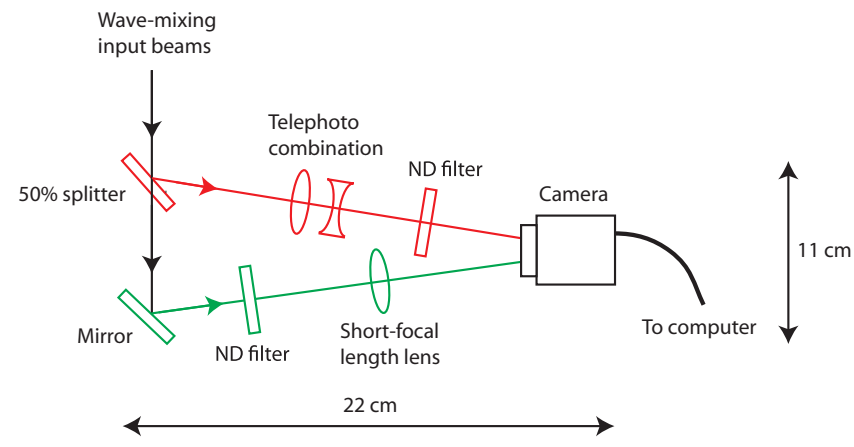

FIG. 1. Schematic diagram of the near-field and far-field viewer. The telephoto combination is composed of a $40 \mathrm{~mm}$ positive lens and a $25 \mathrm{~mm}$ negative lens separated by a distance of $15 \mathrm{~mm}$ such that the effective focal length is infinity. The short focal length lens is a $75 \mathrm{~mm}$ positive lens. The nearfield (green) and far-field (red) images of the beam cross-sections are viewed simultaneously using a single camera.

where $f_{e}$ is the effective focal length, $f_{p}$ is the focal length of the positive lens, $f_{n}$ is the focal length of the negative lens, and $d$ is the distance between the two lenses. We have made a far-field optical system by using a $40 \mathrm{~mm}$ positive lens and a $25 \mathrm{~mm}$ negative lens separated by a distance of $15 \mathrm{~mm}$ in order to give an effective focal length of infinity, as given by Eq. (1). We show a ray diagram of the telephoto combination in Fig. 2. By using this lens combination and a $75 \mathrm{~mm}$ lens for imaging the beams in the near-field, we are able to view the near-field and far-field at the same time using a single camera (DCU223C, Thorlabs GmbH, Germany). To ensure that the camera is not saturated, we placed individual continuously variable neutral density filters (NDL-10C-4, Thorlabs GmbH, Germany) in both the near-field and far-field beam paths to give control of the average power of both sources independently. To check whether the density of the filter altered the beam profiles thereby leading to erroneous alignment, we measured the beam ellipticity as a function of the optical density.

Before we proceed further, we first discuss the chromatic dispersion introduced by the wavelength difference between each pair of laser beams in both the CARS and SFG processes. The wavelength-dependence of the refractive index of a transparent optical material is given approximately by the Sellmeier formula, which is of the form:

$$
n^{2}(\lambda)=A+\frac{B \lambda^{2}}{\lambda^{2}-C}+\frac{D \lambda^{2}}{\lambda^{2}-E},
$$

where $\lambda$ is the wavelength in micrometers and, for the borosilicate glass Schott BK7 used in our lenses here, the coefficients are $A=1.43131380, B=0.84014624, C=1.28897582$ $\times 10^{-2}, D=0.97506873$, and $E=100 .{ }^{16}$ We calculated the refractive indices for our CARS pump and Stokes beams and

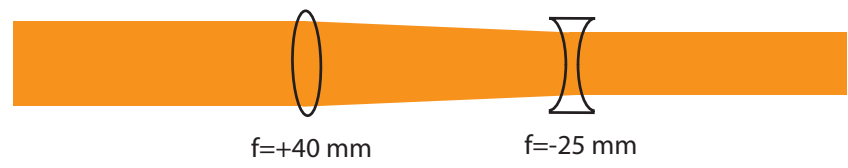

FIG. 2. Ray diagram showing a collimated beam incident on the telephoto combination composed of a $40 \mathrm{~mm}$ positive lens and a $25 \mathrm{~mm}$ negative lens separated by $15 \mathrm{~mm}$. The effective focal length of the combination is infinity.
TABLE I. Table of wavelength-dependent indices of refraction for BK7 and the chromatic dispersion introduced as the wave-mixing beams propagate through the lenses. We compensated for the calculated focus difference ranging from $182 \mu \mathrm{m}$ for the CARS pump and Stokes beams to $891 \mu \mathrm{m}$ for the SFG input beams by using mode-matching optics before the beams are combined.

\begin{tabular}{lcccc}
\hline \hline$\lambda(\mu \mathrm{m})$ & $\mathrm{n}(\lambda)$ & $\begin{array}{c}\mathrm{f}=40 \mathrm{~mm} \\
\text { focus }(\mathrm{mm})\end{array}$ & $\begin{array}{c}\mathrm{f}=-25 \mathrm{~mm} \\
\text { focus }(\mathrm{mm})\end{array}$ & $\begin{array}{c}\mathrm{f}=75 \mathrm{~mm} \\
\text { focus }(\mathrm{mm})\end{array}$ \\
\hline 0.752 & 1.51179 & 40.39313 & -25.24571 & 75.73712 \\
0.957 & 1.50812 & 40.68488 & -25.42805 & 76.28415 \\
1.064 & 1.50664 & 40.80373 & -25.50233 & 76.50699 \\
1.538 & 1.50081 & 41.27873 & -25.79921 & 77.39762 \\
\hline \hline
\end{tabular}

our sum-frequency generation input beams and used these to obtain the focus difference between the beams as they propagate through the lenses.

Comar Instruments specifies a center wavelength of $587 \mathrm{~nm}$ for the focal lengths of the lenses we have used here. At this wavelength, Eq. (2) gives a refractive index of 1.51682 for BK7. We use this refractive index to obtain the radii of curvature of our lenses from the lensmaker's equation: ${ }^{17}$

$$
\frac{1}{f}=(n-1)\left(\frac{1}{R_{1}}-\frac{1}{R_{2}}+\frac{(n-1) d}{n R_{1} R_{2}}\right),
$$

where $f$ is the focal length, $n$ is the refractive index, $R_{1}$ and $R_{2}$ are the radii of curvature, and $d$ is the thickness of the lens. To illustrate, we use our $40 \mathrm{~mm}$ planoconvex lens as an example. The radius of curvature $R_{2}$ for this lens is infinity. Using the refractive index for the center wavelength, we therefore obtain $R_{1}$ to be $20.673 \mathrm{~mm}$ from Eq. (3). Using this value for $R_{1}$, we then calculate the wavelength-dependent focal lengths in Eq. (3) using the refractive indices from Eq. (2). We find that for our CARS pump and Stokes beams of $752 \mathrm{~nm}$ and $957 \mathrm{~nm}$, respectively, there is a $291.75 \mu \mathrm{m}$ focus difference. In the case of our SFG inputs of $1064 \mathrm{~nm}$ and $1538 \mathrm{~nm}$, there is a $475.00 \mu \mathrm{m}$ focus difference. We summarize our calculations for all three lenses in Table I. We have compensated for these focus differences by using mode-matching optics in the individual beam paths. In addition to addressing the dispersion in focal lengths, we also investigated dispersion in astigmatism by varying the centering of the beams on the lenses until the image contrast vanished in the resulting microscope image and quantifying the beam separation as viewed on the alignment device camera.

Because of its extremely small footprint of only $22 \mathrm{~cm}$ $\times 11 \mathrm{~cm} \times 16 \mathrm{~cm}$, our near-field and far-field viewer can be inserted easily into the beam path to check that the beams are coaxial and removed again after any necessary adjustment. We used it thus to align and match the pump and Stokes beams in our homebuilt CARS microscope based on an optical parametric oscillator (OPO) synchronously pumped by a picosecond-pulsed Ti:sapphire laser. ${ }^{18}$ This CARS microscope uses a Ti:sapphire laser output of $752 \mathrm{~nm}$ as the pump beam and an OPO signal with a wavelength range of 920$1200 \mathrm{~nm}$ as the Stokes beam, giving a Raman range of 2430$4960 \mathrm{~cm}^{-1}$ which covers the $\mathrm{C}-\mathrm{H}$ stretching band, making it well-suited for imaging lipids in biological specimens. ${ }^{19}$ The schematic diagram is shown in Fig. 3. We define our near field 


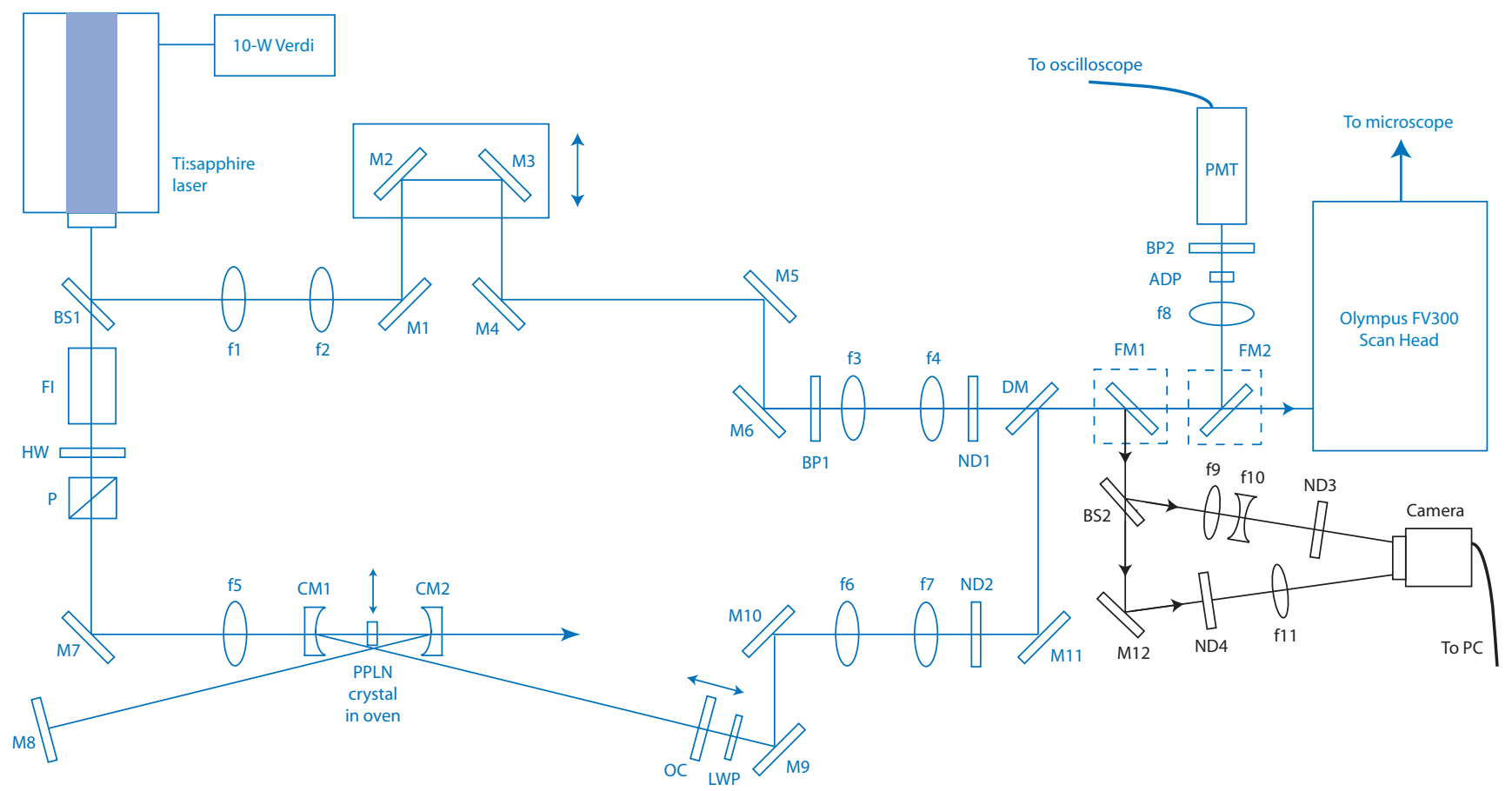

FIG. 3. Schematic diagram of the homebuilt CARS microscope (blue) incorporating the near-field and far-field viewer (black). BS1 $=90 / 10$ beamsplitter, f1-f8 $=$ planoconcave lenses, M1-M11 = highly reflective mirrors for near IR, FI = Faraday isolator, HW = half-wave plate, $\mathrm{P}=$ polarizer, $\mathrm{CM} 1-2=\mathrm{OPO}$ cavity mirrors, PPLN = periodically poled lithium niobate crystal for parametric generation, OC $=$ output coupler, $\mathrm{LWP}=$ long-wave pass filter, $\mathrm{BP} 1=\mathrm{Semrock}$ FF01-769/41 band pass filter, BP2 = Chroma HQ430/50m-2p band pass filter, ND1-2 = neutral density filters, DM = dichroic mirror, FM1-2 = flip-mounted mirrors, $\mathrm{ADP}=$ ammonium dihydrogen phosphate crystal for sum-frequency generation, $\mathrm{PMT}=$ photomultiplier tube.

as the point where the beams are just about to enter the microscope scan head and our far field to be at the microscope specimen plane. We use the pair of mirrors M5 and M6 for the pump beam, and M10 and M11 for the Stokes beam, to aid us in the alignment process. Using our alignment device, achieving spatial overlap and proper matching of beam diameters is straightforward and quickly done, producing a CARS image when the beams are coupled into the microscope scan head. The beam alignment is then further optimized to obtain maximum image contrast as viewed on the microscope.

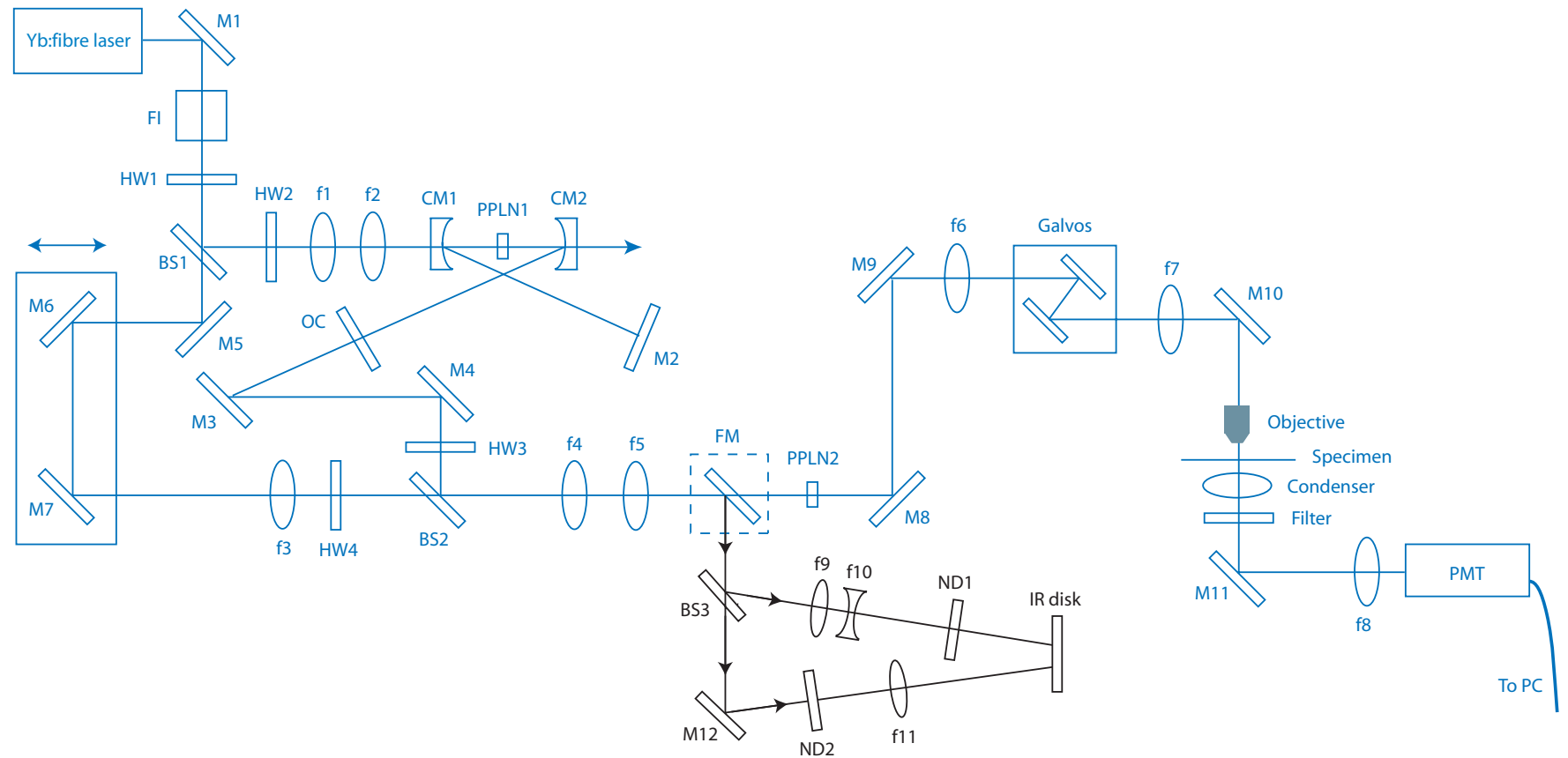

FIG. 4. Schematic diagram of the homebuilt two-photon microscope (blue) incorporating the near-field and far-field viewer (black). M1-M7 $=$ highly reflective mirrors for IR, FI = Faraday isolator, HW1-4 = half-wave plates, BS1 $=50 \%$ splitter, f1-f8 = planoconcave lenses, CM1-2 = OPO cavity mirrors, PPLN1 $=$ periodically poled lithium niobate crystal for parametric generation, $\mathrm{OC}=$ output coupler, PPLN2 $=$ periodically poled lithium niobate crystal for sumfrequency generation, M8-11 = highly reflective mirrors for the visible spectrum, Galvos $=$ galvanometer scanning mirrors, PMT $=$ photomultiplier tube. 


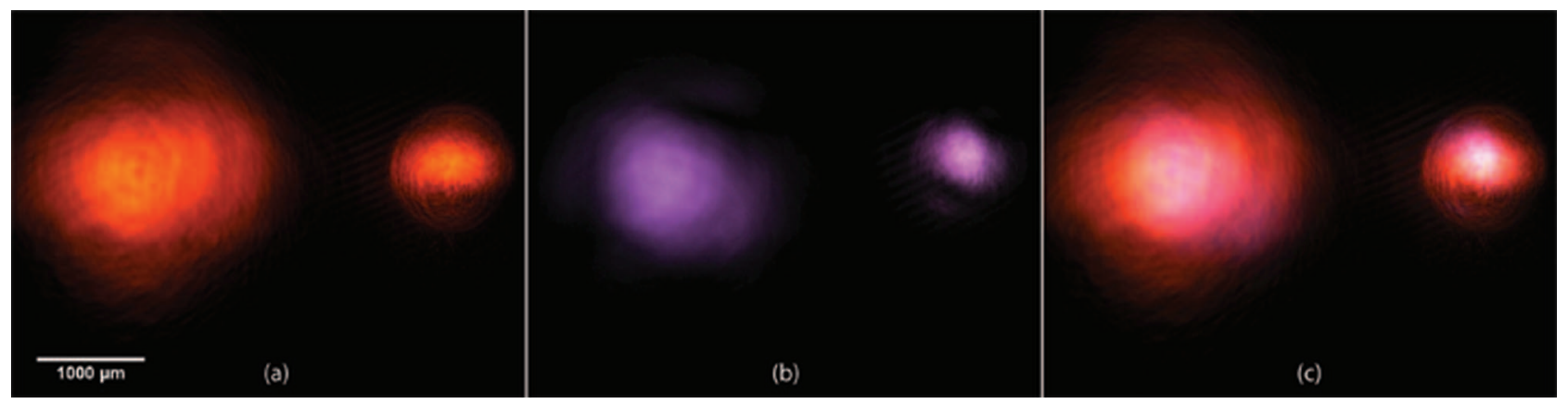

FIG. 5. (a) The Ti:sapphire beam (orange) with the OPO blocked, (b) the OPO beam (violet) with the Ti:sapphire blocked, and (c) the Ti:sapphire and OPO beams both unblocked, showing spatial overlap as viewed on the camera. The far-field is on the left side of each panel while the near-field is on the right.

The device is therefore a useful tool in speeding up the CARS imaging process.

We also used our device to align and match the input beams in our homebuilt SFG-based two-photon microscope, the schematic diagram of which is shown in Fig. 4. To provide the input beams for sum-frequency generation, we used a femtosecond-pulsed Yb-doped fibre laser output of 1064 $\mathrm{nm}$ and an OPO signal with a wavelength range of 1450$1600 \mathrm{~nm}$. As with the CARS microscope, we used the nearfield and far-field viewer to achieve beam overlap and proper matching of beam diameters. Since the laser wavelengths are much longer in this microscope, we used an IR disk (VRC2, Thorlabs GmbH, Germany) in place of the camera to view the beam cross-sections. Achieving spatial overlap and matching of beam diameters is again straightforward, and we generate a sum-frequency output range of $622-636 \mathrm{~nm}$ well-suited for imaging fluorophores with peak two-photon excitation in the ultraviolet region. ${ }^{20}$ The beam alignment is then further optimized to obtain maximum beam power as measured with a power meter. This sub-700 $\mathrm{nm}$ beam is then coupled into a homebuilt laser scanning microscope for two-photon imaging. 21

\section{RESULTS}

We first show our results from the homebuilt CARS microscope. Figure 5 shows the spatially overlapped Ti:sapphire and OPO near-field and far-field beams as viewed on the camera. We found our near-field and far-field viewer sensitive to very small changes in the alignment of either beam, making it highly useful both for initial alignment setting and periodic maintenance. To investigate whether the density of the filters in the beam path altered the beam profiles thereby leading to erroneous alignment, we measured the beam ellipticity as a function of optical density and observed that the neutral density filter has no appreciable effect on the beam profiles. By evaluating the shift in the beam positions on the camera image as the beam alignment is adjusted, we observed that the pump and Stokes beams need to be coaxial to better than $400 \mu \mathrm{rad}$, otherwise the CARS image contrast vanishes. In addition, by varying the centering of the beams on the lenses, we observed that the CARS image contrast vanishes if the beams are offcenter by $50 \mu \mathrm{m}$, corresponding to a center-to-center distance of separation of $380 \mu \mathrm{m}$ between the beams as viewed on the alignment device camera.

We show a sample image from our homebuilt CARS microscope in Fig. 6. We imaged a fresh unstained mouse ear tissue using a pump wavelength of $752 \mathrm{~nm}$ and a Stokes wavelength of $957 \mathrm{~nm}$, resulting in a Raman shift of $2850 \mathrm{~cm}^{-1}$ that coincides with the $\mathrm{C}-\mathrm{H}$ stretch, suitable for imaging lipids in biological specimens. The image shows a cluster of lipid-rich sebaceous glands surrounding a hair follicle. The image size is $512 \times 512$ pixels taken at 1 frame per second. The pump power and Stokes power incident on the specimen were approximately $20 \mathrm{~mW}$ and $40 \mathrm{~mW}$, respectively.

Figure 7 shows a sample image from our homebuilt twophoton fluorescence microscope acquiring images using our sum-frequency-generated laser source. We imaged the radula of the marine snail Littorina, a rasp-like structure used for feeding. ${ }^{22}$ The excitation wavelength is $630 \mathrm{~nm}$, suitable for two-photon excitation of autofluorescence in the ultraviolet region. ${ }^{23}$ The image shows the arrangement of chitinous teeth on the radula ribbon. The image size is $512 \times 512$ pixels taken at 2 frames per second.

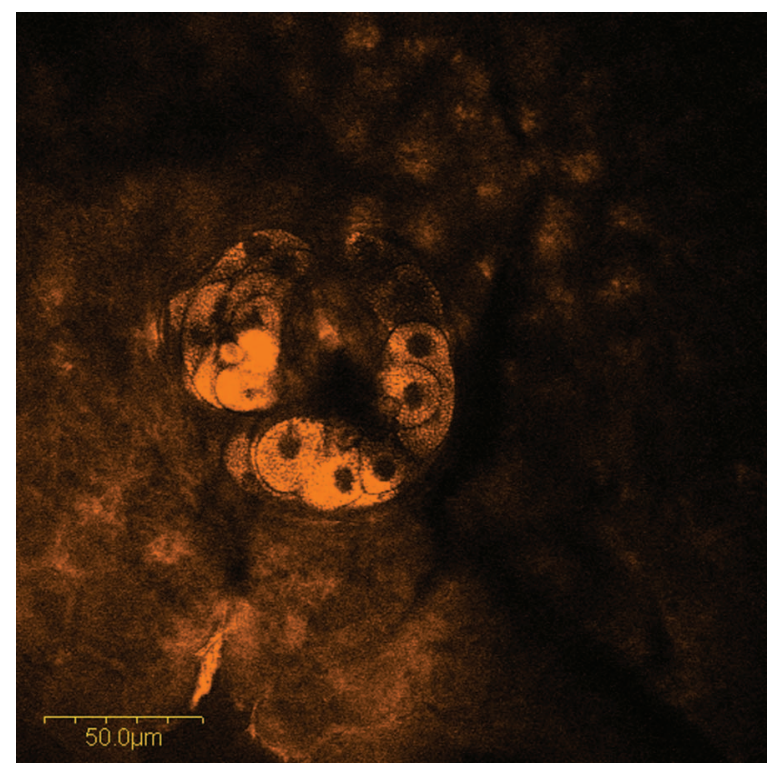

FIG. 6. A CARS image showing a cluster of sebaceous glands in a fresh unstained mouse ear tissue. The image was taken at a Raman shift of $2850 \mathrm{~cm}^{-1}$ to coincide with the $\mathrm{C}-\mathrm{H}$ stretching vibration. 


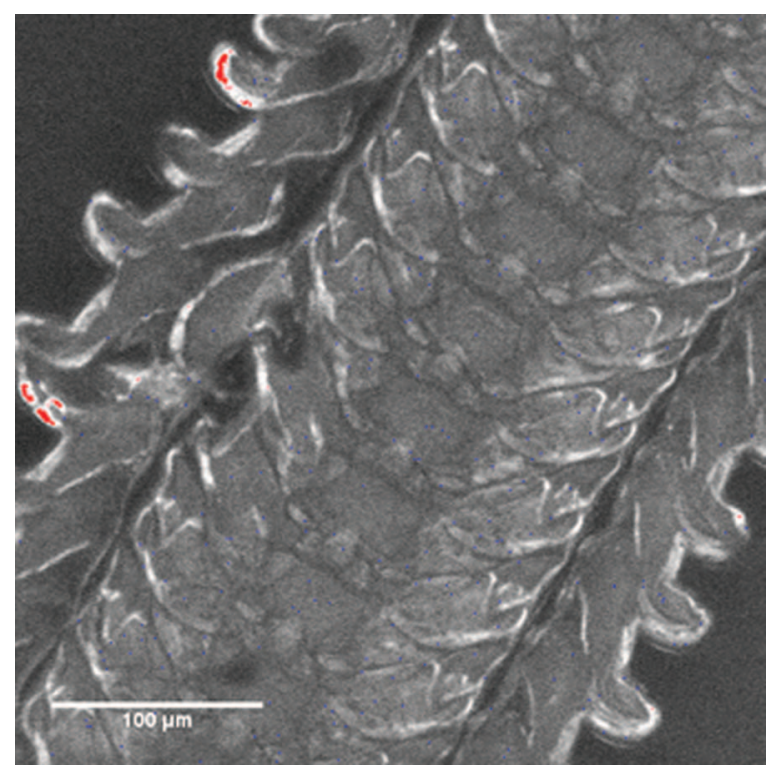

FIG. 7. A two-photon autofluorescence image of snail radula taken with the sum-frequency generation-based fs-pulsed red laser at $630 \mathrm{~nm}$. The arrangement of chitinous teeth on the radula ribbon is clearly visible.

\section{CONCLUSION}

In summary, we have described a simultaneous nearfield and far-field viewer to evaluate spatial overlap in wavemixing experiments. By using a telephoto combination and a short focal length lens, the near-field and far-field beams are viewed simultaneously on a single camera. We have demonstrated the application of the instrument for biomedical imaging by incorporating it in our homebuilt CARS and twophoton fluorescence microscopes.

\section{ACKNOWLEDGMENTS}

We thank Dr. O. Millington for the preparation of the mouse ear specimen. This work was supported by the Scottish Universities Physics Alliance (SUPA), Solus Technologies Limited, and the Engineering and Physical Sciences Research Council (EPSRC).

${ }^{1}$ P. D. Maker and R. W. Terhune, Phys. Rev. 137, A801 (1965).

${ }^{2}$ R. F. Begley, A. B. Harvey, and R. L. Byer, Appl. Phys. Lett. 25, 387 (1974).

${ }^{3}$ M. D. Duncan, J. Reintjes, and T. J. Manuccia, Opt. Lett 7, 350 (1982).

${ }^{4}$ A. Zumbusch, G. R. Holtom, and X. S. Xie, Phys. Rev. Lett. 82, 4142 (1999).

${ }^{5}$ M. Hashimoto, T. Araki, and S. Kawata, Opt. Lett. 25, 1768 (2000).

${ }^{6}$ C. L. Evans, E. O. Potma, M. Puoris'haag, D. Cote, C. P. Lin, and X. S. Xie, Proc. Natl. Acad. Sci. 102, 16807 (2005).

${ }^{7}$ P. F. Curley, A. I. Ferguson, J. G. White, and W. B. Amos, Opt. Quantum Electron. 24, 851 (1992).

${ }^{8}$ S. Huang, A. A. Heikal, and W. W. Webb, Biophys. J. 82, 2811 (2002).

${ }^{9}$ A. Volkmer, J. X. Cheng, and X. S. Xie, Phys. Rev. Lett. 87, 23901 (2001).

${ }^{10}$ T. Hellerer, A. Schiller, G. Jung, and A. Zumbusch, ChemPhysChem 7, 630 (2002).

${ }^{11}$ D. J. Jones, E. O. Potma, J. X. Cheng, B. Burfeindt, Y. Pang, J. Ye, and X. S. Xie, Rev. Sci. Instrum. 73, 2843 (2002).

${ }^{12}$ X. D. Zhu, H. Suhr, and Y. R. Shen, Phys. Rev. B 35, 3047 (1987).

${ }^{13}$ A. Zumbusch, personal communication (2009).

${ }^{14}$ T. J. Weeks and T. R. Huser, J. Vis. Exp. 44, e2085 (2010).

${ }^{15}$ E. Hecht, Optics, 2nd ed. (Addison-Wesley, Reading, Massachusetts, 1987).

${ }^{16}$ G. Ghosh, Appl. Opt. 36, 1540 (1997).

${ }^{17}$ F. A. Jenkins and H. E. White, Fundamentals of Optics, 4th ed. (McGrawHill, New York, 1976).

${ }^{18}$ W. Zhang, M. Parsons, and G. McConnell, Microsc. Res. Tech. 73, 650 (2010).

${ }^{19}$ C. L. Evans and X. S. Xie, Annu. Rev. Anal. Chem. 1, 883 (2008).

${ }^{20}$ G. Norris, Ph.D. dissertation, University of Strathclyde, 2011.

${ }^{21}$ G. Norris, R. Amor, J. Dempster, W. B. Amos, and G. McConnell, J. Microsc. 246, 266 (2012).

${ }^{22}$ D. K. Padilla, Mar. Biol. 90, 103 (1985).

${ }^{23}$ W. Denk, J. H. Strickler, and W. W. Webb, Science 248, 73 (1990). 